\title{
A UNIFIED VIEW OF THE DEDEKIND COMPLETION OF POINTFREE FUNCTION RINGS
}

\author{
JAVIER GUTIÉRREZ GARCÍA, IMANOL MOZO CAROLLO, AND JORGE PICADO
}

\begin{abstract}
We provide the appropriate unifying framework for the various descriptions of the Dedekind completion of the ring $\mathrm{C}(L)$ of continuous real functions on a frame $L$. It is based on suitable Galois connections and a general result about Galois connections, showing once more the ubiquity of (Galois) adjunctions between partially ordered sets and their conceptual simplicity and extent.
\end{abstract}

\section{INTRODUCTION}

This paper takes another look at the Dedekind completion of the ring $\mathrm{C}(L)$ of continuous real functions on a frame $L$. In two previous papers $([7,3])$ we have presented its construction in three different ways, respectively in terms of

(1) partial real functions on $L$,

(2) normal semicontinuous real functions on $L$, and

(3) Hausdorff continuous partial real functions on $L$.

To put them in perspective, we give a brief synopsis of each one:

(1) Recall the frame $\mathfrak{L}(\mathbb{I R})$ of partial real numbers ([7]) defined by generators $(q,-)$ and $(-, q), q \in \mathbb{Q}$, and relations $(\mathrm{R} 1)(q,-)=\bigvee_{p>q}(p,-)$, for every $q \in \mathbb{Q}$,

Date: April 22, 2016.

2010 Mathematics Subject Classification. Primary: 06D22; Secondary: 26A15, 54C30, $54 \mathrm{D} 15$.

Key words and phrases. frame, locale, frame of reals, continuous real function, function ring, order complete, Dedekind completion, scale, normal semicontinuous real function, partial real function, Hausdorff continuous real function, cb-frame.

Research supported by the Ministry of Economy and Competitiveness of Spain (grant MTM2012-37894-C02-02), the University of the Basque Country UPV/EHU (grant GIU12/39) and the Centre for Mathematics of the University of Coimbra (funded by the Portuguese Government through FCT/MEC and co-funded by the European Regional Development Fund through the Partnership Agreement PT2020). I. Mozo Carollo gratefully acknowledges financial assistance from a Predoctoral Fellowship of the Basque Country Government (BFI-2012-262). 
(R2) $(-, q)=\bigvee_{p<q}(-, p)$, for every $p \in \mathbb{Q}$,

(R3) $\bigvee_{q \in \mathbb{Q}}(q,-)=1$,

(R4) $\bigvee_{q \in \mathbb{Q}}(-, q)=1$,

(R5) $(-, q) \wedge(p,-)=0$ whenever $q \leqslant p$.

The class $\operatorname{IC}(L)$ of continuous partial real functions on $L$ is the collection of all frame homomorphisms $\mathfrak{L}(\mathbb{I R}) \rightarrow L$. This is a Dedekind complete lattice containing $\mathrm{C}(L)$. The Dedekind completion of $\mathrm{C}(L)$ inside $\mathrm{IC}(L)$ is given by $\mathrm{C}(L)^{\mathfrak{x}}=\{h \in \mathrm{IC}(L) \mid$ (a) there exist $f, g \in \mathrm{C}(L)$ such that $f \leqslant h \leqslant g$ (b) $h(p,-)^{*} \leqslant h(-, q)$ and $h(-, q)^{*} \leqslant h(p,-)$ for any $p<q$ in $\left.\mathbb{Q}\right\}$.

(2) Recall the frame $\mathfrak{L}(\mathbb{R})$ of real numbers defined by imposing the following further relation to $\mathfrak{L}(\mathbb{R})$ :

(R6) $(p,-) \vee(-, q)=1$ whenever $p<q$.

Let $\mathcal{S}(L)$ denote the frame of sublocales of $L$. The ring $\mathrm{F}(L)$ of general real functions on $L([2])$ is the collection of all frame homomorphisms $\mathfrak{L}(\mathbb{R}) \rightarrow$ $\mathcal{S}(L)$. Of importance here is a special class of lower semicontinuous real functions, called normal [5], which are characterized by the properties $f^{\circ} \in \mathrm{F}(L)$ and $f^{-\circ}=f$, (where $f^{\circ}$ and $f^{-}$denote the lower and upper regularizations of $f$, respectively). The completion of $\mathrm{C}(L)$ is isomorphic with the lattice

$\operatorname{NLSC}^{c b}(L)=\{f \in \mathrm{F}(L) \mid f$ is normal lower semicontinuous and there exist $g, h \in \mathrm{C}(L)$ such that $g \leqslant f \leqslant h\}$.

(3) Recall the ring $\operatorname{IF}(L)$ of general partial real functions on $L$ (i.e. the collection of all frame homomorphisms $\mathfrak{L}(\mathbb{R}) \rightarrow \mathcal{S}(L))$ and its subclasses $\operatorname{IF}^{c b}(L)$ and $\operatorname{IF}^{l b}(L)$ of, respectively, continuously bounded and locally bounded members. An element $f$ in the former is characterized by the property $h_{1} \leqslant$ $f \leqslant h_{2}$ for some $h_{1}, h_{2} \in \mathrm{C}(L)$, whilst in the latter is characterized by the property $\bigvee_{r \in \mathbb{Q}} \overline{f(r,-)}=1=\bigvee_{r \in \mathbb{Q}} \overline{f(-, r)}$. An $f \in \mathrm{IF}^{l b}(L)$ is Hausdorff continuous if $f \in \operatorname{IC}(L)$, i.e., $f(p,-)$ and $f(-, q)$ are closed sublocales for every $p, q \in \mathbb{Q}, f^{\circ-}=f^{-}$and $f^{-\circ}=f^{\circ}$. Denoting by $\mathrm{H}(L)$ the collection of all Hausdorff continuous partial real functions on $L$, the completion of $\mathrm{C}(L)$ is isomorphic with

$$
\mathrm{H}^{c b}(L)=\mathrm{H}(L) \cap \mathrm{IF}^{c b}(L) .
$$

The purpose of this paper is to present a unified view of the three representations above in a single general diagram of (Galois) adjunctions, based on a suitable collection of scales in $L$. We construct three adequate Galois connections between this collection and each one of the three representing 
lattices above. Then, the fact that they all describe the Dedekind completion of $\mathrm{C}(L)$ will follow from an easy general fact about Galois connections.

As a general reference for frames and locales we suggest [8]. We refer to [1] for specific facts about the frame of reals and the corresponding ring of continuous real-valued functions on a frame $L$, and to [2] for the ring $\mathrm{F}(L)$ of general real functions on $L$. For the details about the three constructions mentioned above, the reader should please consult our previous [7] (for the first) and [3] (for the other two). The notation used in the present paper without explanation is that of those preceding papers.

\section{Dedekind completions and Galois Connections}

Recall (see, e.g., [8, Appendix I.5]) that two monotone maps

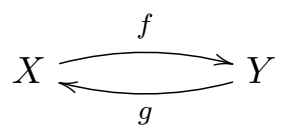

between posets $X$ and $Y$ are Galois adjoint (or are in a Galois connection) if

$$
\forall x \in X, \forall y \in Y, \quad f(x) \leqslant y \quad \Longleftrightarrow \quad x \leqslant g(y) .
$$

In this situation, $f$ is said to be a left adjoint of $g$ (and $g$ is a right adjoint of $f$ ), denoted briefly as $f \dashv g$. Equivalently, monotone $f: X \rightarrow Y$ and $g: Y \rightarrow X$ are adjoint if and only if

$$
\forall x \in X, \forall y \in Y, \quad f(g(y)) \leqslant y \quad \text { and } \quad x \leqslant g(f(x)) .
$$

Left Galois adjoints preserve all suprema that exist in $X$, and the right ones preserve infima. If $X$ and $Y$ are complete lattices, then a monotone map $f: X \rightarrow Y$ is a left (resp. right) adjoint if and only if it preserves all suprema (resp. infima).

We follow [9, Section 1.3] for the terminology on completions of a poset. We recall from there that a completion of $P$ is a pair $(C, \varphi)$ where $C$ is a complete lattice and $\varphi: P \rightarrow C$ is a join- and meet-dense embedding (that is, each element of $C$ is a join of elements from $\varphi[P]$, and dually each element of $C$ is a meet of elements from $\varphi[P])$.

A poset $P=(P, \leqslant)$ is Dedekind (order) complete (or conditionally complete) if every non-void subset $A$ of $P$ which is bounded from above has a supremum in $P$ (and then, in particular, every non-void subset $B$ of $P$ which is bounded from below will have an infimum in $P$ ). Of course, being complete is equivalent to being Dedekind complete plus the existence of top and bottom elements. A Dedekind completion (or conditional completion) of $P$ 
is a join- and meet-dense embedding $\varphi: P \rightarrow D(P)$ in a Dedekind complete poset $D(P)$.

Finally, a poset $X$ is self-dual if there exists a dual-order isomorphism, i.e. an antitone and bijective $\varphi: X \rightarrow X$ with antitone inverse.

Theorem 1.1. Let $X$ be a self-dual poset, $Y$ a Dedekind complete lattice and

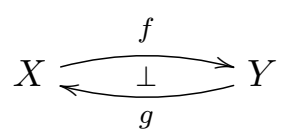

a Galois connection such that ${ }^{1} g \circ f=1_{X}$. Then $X$ is Dedekind complete.

Moreover, if $\varphi: P \rightarrow Y$ is a Dedekind completion of a poset $P$, then the inclusion $\iota:(g \circ \varphi)[P] \rightarrow X$ is a Dedekind completion of the poset $(g \circ \varphi)[P]$ whenever $(g \circ \varphi)[P]$ is also self-dual as a subposet of $X$ by the restriction of the dual-order isomorphism of $X$.

Proof. Let $\varnothing \neq S \subseteq X$ be bounded from below by some $x \in X$. Since $f$ is order-preserving, one has that $f[S]$ is bounded from below by $f(x)$. As $Y$ is Dedekind complete, the meet $\bigwedge f[S]$ does exist in $Y$. Then $g(\bigwedge f[S])=$ $\bigwedge(g \circ f)[S]=\bigwedge S$. Hence, $X$ is closed under non-void bounded infima. Since $X$ is self-dual, we may conclude that it is also closed under bounded suprema and therefore, that it is Dedekind complete.

In order to check that the inclusion $\iota:(g \circ \varphi)[P] \rightarrow X$ is a Dedekind completion of $(g \circ \varphi)[P]$, consider an arbitrary $x \in X$. Since $\varphi: P \rightarrow Y$ is a Dedekind completion of $P$ we have $f(x)=\bigwedge\{\varphi(p) \mid p \in P$ and $f(x) \leqslant \varphi(p)\}$. Consequently,

$$
\begin{aligned}
x=g(f(x)) & =\bigwedge\{g(\varphi(p)) \mid p \in P \text { and } f(x) \leqslant \varphi(p)\} \\
& =\bigwedge\{g(\varphi(p)) \mid p \in P \text { and } x \leqslant g(\varphi(p))\} .
\end{aligned}
$$

Hence $(g \circ \varphi)[P]$ is meet-dense in $X$. By self-duality, it is also join-dense.

\section{SCALES}

In what follows $L$ will always denote a frame.

There is a useful way of specifying continuous real functions on $L$ with the help of the so-called scales. This is explained in detail in [4] or [6]. Here we just recall that a scale in $L$ is a map $\sigma: \mathbb{Q} \rightarrow L$ such that

(1) $\sigma(q)<\sigma(p)$ whenever $p<q$, and

(2) $\bigvee_{q \in \mathbb{Q}} \sigma(q)=1=\bigvee_{q \in \mathbb{Q}} \sigma(q)^{*}$.

\footnotetext{
${ }^{1}$ Galois connections $f \dashv g$ such that $g$ is a left inverse of $f$ are sometimes named Galois injections.
} 
For each scale $\sigma$ the formulas

$$
f_{\sigma}(r,-)=\bigvee_{q>r} \sigma(q) \quad \text { and } \quad f_{\sigma}(-, s)=\bigvee_{q<s} \sigma(q)^{*} \quad \text { for all } r, s \in \mathbb{Q}
$$

determine a continuous real function $f_{\sigma}: \mathfrak{L}(\mathbb{R}) \rightarrow L$. Conversely, each continuous real function $f: \mathfrak{L}(\mathbb{R}) \rightarrow L$ yields a scale $\sigma_{f}: \mathbb{Q} \rightarrow L$ defined by

$$
\sigma_{f}(q)=f(q,-) \quad \text { for all } q \in \mathbb{Q}
$$

and, by formulas (1.1), the scale $\sigma_{f}$ induces the original $f$.

We will denote by $\operatorname{Sc}(L)$ the set of all scales on $L$. This set is partially ordered by

$$
\sigma \leqslant \gamma \quad \equiv \sigma(q) \leqslant \gamma(q) \text { for every } q \in \mathbb{Q} \text {. }
$$

(Note that $\sigma \leqslant \gamma$ implies $f_{\sigma} \leqslant f_{\gamma}$ and, conversely, $f \leqslant g$ implies $\sigma_{f} \leqslant \gamma_{f}$ ).

We shall also need the following weaker version of a scale: a generalized scale in $L$ is just an antitone map $\sigma: \mathbb{Q} \rightarrow L$ such that

$$
\bigvee_{q \in \mathbb{Q}} \sigma(q)=1=\bigvee_{q \in \mathbb{Q}} \sigma(q)^{*} .
$$

We will denote by $\operatorname{GSc}(L)$ the set of all generalized scales in $L$. Note that a scale $\sigma$ is always antitone and, consequently, $\operatorname{Sc}(L) \subseteq \operatorname{GSc}(L)$. Of course, the partial order in $\operatorname{Sc}(L)$ can be naturally extended to $\operatorname{GSc}(L)$.

Given a generalized scale $\sigma$, there is also the generalized scale $\sigma^{* *}$ defined by $\sigma^{* *}(q)=\sigma(q)^{* *}$ for all $q \in \mathbb{Q}$. Evidently, the correspondence $\sigma \mapsto \sigma^{* *}$ establishes an order-preserving map in $\operatorname{GSc}(L)$. Moreover, if $\sigma$ is a scale, then $\sigma^{* *}$ and $\sigma$ induce the same continuous real function via formulas (1.1).

We say that a generalized scale $\sigma$ is regular if all its images $\sigma(q)$ are regular elements of $L$, that is, $\sigma(q)=\sigma(q)^{* *}$. In other words, $\sigma$ is regular if and only if $\sigma=\sigma^{* *}$. We will denote by $\operatorname{RegGSc}(L)$ and $\operatorname{RegSc}(L)$ the sets of regular generalized scales and regular scales, respectively.

Remarks 2.1. (1) There is a dual-order isomorphism

$$
-(\cdot): \operatorname{RegGSc}(L) \rightarrow \operatorname{RegGSc}(L)
$$

defined by

$$
(-\sigma)(q)=\sigma(-q)^{*} \quad \text { for all } q \in \mathbb{Q} .
$$

Its restriction to $\operatorname{RegSc}(L)$ yields a dual-order isomorphism between $\operatorname{RegSc}(L)$ and $\operatorname{RegSc}(L)$, that is, $\operatorname{RegSc}(L)$ is a self-dual poset.

(2) It is also worth mentioning that for any generalized scale $\sigma$,

$$
\sigma^{* *}=\min \{\gamma \in \operatorname{RegGSc}(L) \mid \sigma \leqslant \gamma\} .
$$




\section{Scales And DedeKind Completions}

Proposition 3.1. The poset $\operatorname{GSc}(L)$ is Dedekind complete. Specifically, we have:

(1) Given any non-void $\left\{\sigma_{i}\right\}_{i \in I} \subseteq \operatorname{GSc}(L)$ and $\sigma \in \operatorname{GSc}(L)$ such that $\sigma_{i} \leqslant \sigma$ for all $i \in I$, the supremum of $\left\{\sigma_{i}\right\}_{i \in I}$ in $\operatorname{GSc}(L)$ is given by

$$
\left(\bigvee^{\operatorname{GSc}(L)_{i \in I}} \sigma_{i}\right)(q)=\bigvee_{i \in I} \sigma_{i}(q) \quad \text { for every } q \in \mathbb{Q}
$$

(2) Given any non-void $\left\{\sigma_{i}\right\}_{i \in I} \subseteq \operatorname{GSc}(L)$ and $\sigma \in \operatorname{GSc}(L)$ such that $\sigma \leqslant \sigma_{i}$ for all $i \in I$, the infimum of $\left\{\sigma_{i}\right\}_{i \in I}$ in $\operatorname{GSc}(L)$ is given by

$$
\left(\bigwedge^{\operatorname{GSc}(L)_{i \in I}} \sigma_{i}\right)(q)=\bigwedge_{i \in I} \sigma_{i}(q) \quad \text { for every } q \in \mathbb{Q}
$$

Proof. (1) First note that the map $\sigma_{\vee}: \mathbb{Q} \rightarrow L$, given by $\sigma_{\vee}(q)=\bigvee_{i \in I} \sigma_{i}(q)$ for every $q \in \mathbb{Q}$, is obviously antitone and that

$$
\bigvee_{q \in \mathbb{Q}} \sigma_{\vee}(q)=\bigvee_{q \in \mathbb{Q}} \bigvee_{i \in I} \sigma_{i}(q)=\bigvee_{i \in I} \bigvee_{q \in \mathbb{Q}} \sigma_{i}(q)=1
$$

and

$$
\bigvee_{q \in \mathbb{Q}} \sigma_{\vee}(q)^{*}=\bigvee_{q \in \mathbb{Q}}\left(\bigvee_{i \in I} \sigma_{i}(q)\right)^{*} \geqslant \bigvee_{q \in \mathbb{Q}} \sigma(q)^{*}=1
$$

Therefore, $\sigma_{\vee}$ is a generalized scale on $L$. In order to check that $\sigma_{\vee}$ is actually the supremum of $\left\{\sigma_{i}\right\}_{i \in I}$ in $\operatorname{GSc}(L)$, let $\sigma^{\prime} \in \operatorname{GSc}(L)$ be such that $\sigma_{i} \leqslant \sigma^{\prime}$ for every $i \in I$. Then $\sigma_{\vee}(q)=\bigvee_{i \in I} \sigma_{i}(q) \leqslant \sigma^{\prime}(q)$ for all $q \in \mathbb{Q}$.

(2) Analogously, one has that the map $\sigma_{\wedge}: \mathbb{Q} \rightarrow L$, given by $\sigma_{\wedge}(q)=$ $\bigwedge_{i \in I} \sigma_{i}(q)$ for every $q \in \mathbb{Q}$, is also antitone and that

$$
\bigvee_{q \in \mathbb{Q}} \sigma_{\wedge}(q)=\bigvee_{q \in \mathbb{Q}} \bigwedge_{i \in I} \sigma_{i}(q) \geqslant \bigvee_{q \in \mathbb{Q}} \sigma(q)=1
$$

Fixing an $i_{0} \in I$, we get also

$$
\bigvee_{q \in \mathbb{Q}} \sigma_{\wedge}(q)^{*}=\bigvee_{q \in \mathbb{Q}}\left(\bigwedge_{i \in I} \sigma_{i}(q)\right)^{*} \geqslant \bigvee_{q \in \mathbb{Q}} \sigma_{i_{0}}(q)^{*}=1 .
$$

Moreover, for any $\sigma^{\prime} \in \operatorname{GSc}(L)$ such that $\sigma^{\prime} \leqslant \sigma_{i}$ for all $i \in I$, we have $\sigma_{\wedge}(q)=\bigwedge_{i \in I} \sigma_{i}(q) \geqslant \sigma^{\prime}(q)$ for all $q \in \mathbb{Q}$.

Next result is an immediate consequence of the preceding proposition and Remark 2.1(2).

Corollary 3.2. The poset $\operatorname{RegGSc}(L)$ is Dedekind complete. Specifically, given any non-void $\left\{\sigma_{i}\right\}_{i \in I} \subseteq \operatorname{RegGSc}(L)$ and any $\sigma \in \operatorname{RegGSc}(L)$ such that 
$\sigma_{i} \leqslant \sigma$ for all $i \in I$, the supremum of $\left\{\sigma_{i}\right\}_{i \in I}$ in $\operatorname{Reg} \operatorname{GSc}(L)$ is given by

$$
\left(\bigvee_{i \in I}^{\operatorname{GSc}(L)} \sigma_{i}\right)^{* *}
$$

Proposition 3.3. Let $L$ be a completely regular frame and let $\sigma \in \operatorname{GSc}(L)$ be such that $\{\gamma \in \operatorname{Sc}(L) \mid \gamma \leqslant \sigma\} \neq \varnothing$. Then

$$
\sigma=\bigvee^{\operatorname{GSc}(L)}\{\gamma \in \operatorname{Sc}(L) \mid \gamma \leqslant \sigma\}
$$

Proof. Let $\Gamma=\{\gamma \in \operatorname{Sc}(L) \mid \gamma \leqslant \sigma\} \neq \varnothing$. Since GSc $(L)$ is Dedekind complete, the supremum of $\Gamma$ in $\operatorname{GSc}(L)$ does exist. We only need to prove that

$$
\bigvee^{\operatorname{GSc}(L)} \Gamma \geqslant \sigma .
$$

(since the reverse inequality is obvious).

For this purpose, let us fix a $q \in \mathbb{Q}$ and an $a \in L$ such that $a \ll \sigma(q)$. This means that there exists a family $\left\{c_{r} \in L \mid r \in \mathbb{Q} \cap[0,1]\right\}$ such that $a \leqslant c_{0}$, $c_{1} \leqslant \sigma(q)$ and $c_{r}<c_{s}$ whenever $r<s$. Furthermore, consider a dual-order isomorphism $^{2}$

$$
\psi_{q}: \mathbb{Q} \cap(-\infty, q] \rightarrow \mathbb{Q} \cap[0,1) .
$$

Then, for each $\gamma \in \Gamma$ define the mapping $\gamma_{q, a}: \mathbb{Q} \rightarrow L$ by

$$
\gamma_{q, a}(r)= \begin{cases}\gamma(r) & \text { if } r>q \\ \gamma(r) \vee c_{\psi_{q}(r)} & \text { if } r \leqslant q\end{cases}
$$

Each $\gamma_{q, a}$ is clearly antitone and $\bigvee_{r \in \mathbb{Q}} \gamma_{q, a}(r) \geqslant \bigvee_{r \in \mathbb{Q}} \gamma(r)=1$. Further, note that $\gamma_{q, a}(r) \leqslant \sigma(r)$ for all $r \in \mathbb{Q}$ and, consequently, $\bigvee_{r \in \mathbb{Q}} \gamma_{q, a}(r)^{*} \geqslant$ $\bigvee_{r \in \mathbb{Q}} \sigma(r)^{*}=1$. Therefore $\gamma_{q, a}$ is a generalized scale such that $\gamma_{q, a} \leqslant \sigma$. Finally, for any $r<s$ in $\mathbb{Q}$, one has $\gamma(s)<\gamma(r)$ and $c_{\psi_{q}(s)}<c_{\psi_{q}(r)}$, since $\psi_{q}(s)<\psi_{q}(r)$. Thus $\gamma_{q, a}(s)<\gamma_{q, a}(r)$. Consequently, $\gamma_{q, a}$ is a scale and we conclude that $\gamma_{q, a} \in \Gamma$.

In conclusion, by the complete regularity of $L$, we have

$$
\left(\bigvee^{\mathrm{GSc}(L)} \Gamma\right)(q) \geqslant \bigvee_{a<\sigma(q)} \gamma_{q, a}(q)=\bigvee_{a<\sigma(q)} \gamma(q) \vee c_{0} \geqslant \bigvee_{a<\sigma(q)} a=\sigma(q)
$$

Now, let us define a regular generalized scale $\sigma$ to be continuously bounded whenever there exist $\gamma, \delta \in \operatorname{RegSc}(L)$ such that $\gamma \leqslant \sigma \leqslant \delta$. We will denote by $\operatorname{RegGSc}^{c b}(L)$ the collection of all continuously bounded and regular generalized scales.

\footnotetext{
${ }^{2}$ One may take, for instance, the map given by $\psi_{q}(r)=\frac{(r-q)^{2}}{(r-q)^{2}+1}$.
} 
Corollary 3.4. For any completely regular frame $L$, the poset $\operatorname{RegSc}(L)$ is join- and meet-dense in $\operatorname{RegGSc}^{c b}(L)$.

Proof. The fact that $\operatorname{RegSc}(L)$ is join-dense in $\operatorname{RegGSc}(L)^{c b}$ follows immediately from Proposition 3.3 and Remark 2.1 (2). Then, by Remark 2.1 (1), $\operatorname{RegSc}(L)$ is also meet-dense in $\operatorname{RegGSc}(L)^{c b}$.

Corollary 3.5. For any completely regular frame $L$, the inclusion

$$
\iota: \operatorname{RegSc}(L) \rightarrow \operatorname{RegGSc}{ }^{c b}(L)
$$

is a Dedekind completion of $\operatorname{RegSc}(L)$.

\section{Galois Connections and the Unified Picture}

We need first to recall some basic facts about the structure of the sublocale lattice $\mathcal{S}(L)$.

A sublocale $S$ of a frame $L$ is a subset $S \subseteq L$ satisfying

(S1) for every $A \subseteq S, \bigwedge A$ is in $S$, and

(S2) for every $s \in S$ and every $x \in L, x \rightarrow s$ is in $S$.

The lattice of all sublocales constitutes a co-frame (i.e., the dual of a frame) with the order given by inclusion, meet coinciding with the intersection and the join given by $\bigvee S_{i}=\left\{\bigwedge M \mid M \subseteq \bigcup S_{i}\right\}$; the top is $L$ and the bottom is the set $\{1\}$. We make this co-frame into a frame $\mathcal{S}(L)$ just by considering the dual ordering: $S_{1} \leqslant S_{2}$ iff $S_{2} \subseteq S_{1}$. Thus, $\{1\}$ is the top and $L$ is the bottom in $\mathcal{S}(L)$ that we simply denote by 1 and 0 , respectively.

For any $a \in L$, the sets $\mathfrak{c}(a)=\uparrow a$ and $\mathfrak{o}(a)=\{a \rightarrow b \mid b \in L\}$ are the closed and open sublocales of $L$, respectively. They are complements of each other in $\mathcal{S}(L)$. Furthermore, the map $a \mapsto \mathfrak{c}(a)$ is a frame embedding $L \hookrightarrow \mathcal{S}(L)$ providing an isomorphism $\mathfrak{c}$ between $L$ and the subframe $\mathfrak{c} L$ of $\mathcal{S}(L)$ consisting of all closed sublocales. Since the pseudocomplement $a^{*}$ of each $a \in L$ satisfies the identity $a \wedge a^{*}=0$, then $\mathfrak{o}(a) \geqslant \mathfrak{c}\left(a^{*}\right)$ for any $a \in L$.

On the other hand, denoting by $\mathfrak{o} L$ the subframe of $\mathcal{S}(L)$ generated by all $\mathfrak{o}(a)$, the correspondence $a \mapsto \mathfrak{o}(a)$ establishes a dual-order embedding $L \rightarrow \mathfrak{o} L$.

Since we work in the dual lattice $\mathcal{S}(L)$ of the sublocale lattice, the closure (resp. interior) of a sublocale $S$ in $\mathcal{S}(L)$ is the largest closed sublocale contained in $S$, that is, $\bar{S}=\bigvee\{\mathfrak{c}(a) \mid \mathfrak{c}(a) \leqslant S\}$ (resp. the smallest open sublocale containing $S$, that is, $\left.S^{\circ}=\bigwedge\{\mathfrak{o}(a) \mid S \leqslant \mathfrak{o}(a)\}\right)$. Hence, we should not forget that $\bar{S} \leqslant S \leqslant S^{\circ}$. We also recall that $\mathfrak{c}(a)^{\circ}=\mathfrak{o}\left(a^{*}\right)$ and $\overline{\mathfrak{o}(a)}=\mathfrak{c}\left(a^{*}\right)$ (see [8, III.6 and III.8]). 
Now, let us define three mappings

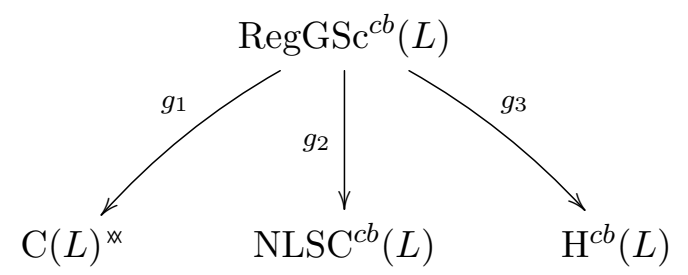

as follows:

For each $\sigma \in \operatorname{RegGSc}^{c b}(L)$,

- $g_{1}(\sigma): \mathfrak{L}(\mathbb{I R}) \rightarrow L$ is defined on generators by

$$
g_{1}(\sigma)(p,-)=\bigvee_{r>p} \sigma(r) \quad \text { and } \quad g_{1}(\sigma)(-, q)=\bigvee_{s<q} \sigma(s)^{*} ;
$$

- $g_{2}(\sigma): \mathfrak{L}(\mathbb{R}) \rightarrow \mathcal{S}(L)$ is defined on generators by

$$
g_{2}(\sigma)(p,-)=\bigvee_{r>p} \mathfrak{c}(\sigma(r)) \quad \text { and } \quad g_{2}(\sigma)(-, q)=\bigvee_{s<q} \mathfrak{o}(\sigma(s)) ;
$$

- $g_{3}(\sigma): \mathfrak{L}(\mathbb{I R}) \rightarrow \mathcal{S}(L)$ is defined on generators by

$$
g_{3}(\sigma)(p,-)=\bigvee_{r>p} \mathfrak{c}(\sigma(r)) \quad \text { and } \quad g_{3}(\sigma)(-, q)=\bigvee_{s<q} \mathfrak{c}\left(\sigma(s)^{*}\right)
$$

In order to confirm that $g_{1}, g_{2}$ and $g_{3}$ are well- defined, we need to check that $g_{1}(\sigma) \in \mathrm{C}(L)^{x}, g_{2}(\sigma) \in \mathrm{NLSC}^{c b}(L)$ and $g_{3}(\sigma) \in \mathrm{H}^{c b}(L)$ :

$\left(g_{1}\right)$ : First, $g_{1}(\sigma)$ is a frame homomorphism, that is, it turns relations (R1)-(R5) into identities in $L$ : The cases (R1) and (R2) are obvious by the definition of $g_{1}(\sigma)$ and the cases (R3) and (R4) follow from the fact that $\sigma$ is a generalized scale. In order to check (R5), let $q \leqslant p$ in $\mathbb{Q}$. We have

$$
g_{1}(\sigma)(-, q) \wedge g_{1}(\sigma)(p,-)=\bigvee_{s<q} \sigma(s)^{*} \wedge \bigvee_{r>p} \sigma(r) \leqslant \sigma(q)^{*} \wedge \sigma(q)=0
$$

Hence, $g_{1}(\sigma)$ is a continuous partial real function on $L$.

Finally, we need to show that $g_{1}(\sigma)$ is indeed in $\mathrm{C}(L)^{\mathrm{x}}$. Of course, $g_{1}$ is order-preserving and it maps regular scales into continuous real functions. Consequently, as $\sigma$ is continuously bounded, $g_{1}(\sigma)$ is also continuously bounded. Furthermore, given $p<q$ in $\mathbb{Q}$, let $t \in \mathbb{Q}$ such that $p<t<q$. Then

$$
g_{1}(\sigma)(p,-)^{*}=\left(\bigvee_{r>p} \sigma(r)\right)^{*}=\bigwedge_{r>p} \sigma(r)^{*} \leqslant \sigma(t)^{*} \leqslant \bigvee_{s<q} \sigma(s)^{*}=g_{1}(\sigma)(-, q) \text {. }
$$

Dually,

$$
\begin{aligned}
g_{1}(\sigma)(-, q)^{*} & =\left(\bigvee_{s<q} \sigma(s)^{*}\right)^{*}=\bigwedge_{s<q} \sigma(s)^{* *}=\bigwedge_{s<q} \sigma(s) \\
& \leqslant \sigma(t) \leqslant \bigvee_{r>p} \sigma(r)=g_{1}(\sigma)(p,-) .
\end{aligned}
$$


$\left(g_{2}\right)$ : Now, we need to check that $g_{2}(\sigma)$ turns relations $(\mathrm{R} 1)-(\mathrm{R} 6)$ into identities in $\mathcal{S}(L)$. As for $g_{1},(\mathrm{R} 1),(\mathrm{R} 2)$ and (R3) are obvious and (R5) may be proved in a similar way. Regarding (R4), we have

$$
\begin{aligned}
\bigvee_{q \in \mathbb{Q}} g_{2}(\sigma)(-, q) & =\bigvee_{q \in \mathbb{Q}} \bigvee_{s<q} \mathfrak{o}(\sigma(s))=\bigvee_{s \in \mathbb{Q}} \mathfrak{o}(\sigma(s)) \geqslant \bigvee_{s \in \mathbb{Q}} \mathfrak{c}\left(\sigma(s)^{*}\right) \\
& =\mathfrak{c}\left(\bigvee_{s \in \mathbb{Q}} \sigma(s)^{*}\right)=1 .
\end{aligned}
$$

Finally, in order to check (R6), let $p<q$ in $\mathbb{Q}$ and consider $t \in \mathbb{Q}$ such that $p<t<q$. Then

$g_{2}(\sigma)(p,-) \vee g_{2}(\sigma)(-, q)=\bigvee_{r>p} \mathfrak{c}(\sigma(r)) \vee \bigvee_{s<q} \mathfrak{o}(\sigma(s)) \geqslant \mathfrak{c}(\sigma(t)) \vee \mathfrak{o}(\sigma(t))=1$

It remains to show that $g_{2}(\sigma)$ belongs to $\operatorname{NLSC}^{c b}(L)$. Since $g_{2}(\sigma)$ is clearly lower semicontinuous (by definition) and continuously bounded, it suffices to prove that $g_{2}(\sigma)^{-\circ} \leqslant g_{2}(\sigma)$. Recall from [3, Lemma 4.8] that

$$
g_{2}(\sigma)^{-\circ}(p,-)=\bigvee_{r>p} \overline{g_{2}(\sigma)(r,-)^{\circ}} \quad \text { and } \quad g_{2}(\sigma)^{-\circ}(-, q)=\bigvee_{s<q}\left(\overline{g_{2}(\sigma)(-, s)}\right)^{\circ}
$$

for each $p, q \in \mathbb{Q}$. Therefore,

$$
\begin{aligned}
g_{2}(\sigma)^{-\circ}(p,-) & =\bigvee_{r>p} \overline{\left(\bigvee_{s>r} \mathfrak{c}(\sigma(s))\right)^{\circ}} \leqslant \bigvee_{r>p} \overline{\mathfrak{c}(\sigma(r))^{\circ}}=\bigvee_{r>p} \mathfrak{c}\left(\sigma(r)^{* *}\right) \\
& =\bigvee_{r>p} \mathfrak{c}(\sigma(r))=g_{2}(\sigma)(p,-)
\end{aligned}
$$

for every $p \in \mathbb{Q}$, from which it follows that $g_{2}(\sigma)^{-\circ} \leqslant g_{2}(\sigma)$.

$\left(g_{3}\right)$ : The fact that each $g_{3}(\sigma)$ is a frame homomorphism follows immediately from the case of $g_{1}$, by the isomorphism between $L$ and $\mathfrak{c} L$. Finally, $g_{3}(\sigma) \in \mathrm{H}^{c b}(L)$. Indeed, it obviously belongs to $\mathrm{IC}(L)$. It remains to check that $g_{3}^{-\circ}=g_{3}^{\circ}$ and $g_{3}^{\circ-}=g_{3}^{-}$but this can be done in a way similar to the previous case so we omit the details.

Proposition 4.1. Each mapping $g_{1}, g_{2}$ and $g_{3}$ is the right Galois map in a Galois adjoint pair that satisfy the conditions of Theorem 1.1. Moreover, for any completely regular frame $L$ and the completion

$$
\iota: \operatorname{RegSc}(L) \rightarrow \operatorname{RegGSc}{ }^{c b}(L)
$$

given by Corollary 3.5,

$$
\left(g_{i} \circ \iota\right)[\operatorname{RegSc}(L)]=\mathrm{C}(L) \quad(i=1,2,3) .
$$


Proof. $\left(f_{1}\right)$ : Let $f_{1}: \mathrm{C}(L)^{\text {w }} \rightarrow \operatorname{RegGSc}{ }^{c b}(L)$ be defined by $f_{1}(h)(q)=h(q,-)^{* *}$ for each $h \in \mathrm{C}(L)^{* x}$ and $q \in \mathbb{Q}$. Obviously, $f_{1}$ is order-preserving and $g_{1} \circ f_{1}=1_{\mathrm{C}(L)}$. On the other hand, for each $\sigma \in \operatorname{RegGSc}^{c b}(L)$ we have

$$
f_{1}\left(g_{1}(\sigma)\right)(q)=g_{1}(\sigma)(q,-)^{* *}=\left(\bigvee_{p>q} \sigma(p)\right)^{* *} \leqslant \sigma(q)^{* *}=\sigma(q)
$$

for all $q \in \mathbb{Q}$, that is, $f_{1} \circ g_{1} \leqslant 1_{\operatorname{RegGSc}^{c b}(L)}$. Hence $f_{1} \dashv g_{1}$.

Moreover, $\left(g_{1} \circ \iota\right)[\operatorname{RegSc}(L)]=g_{1}[\operatorname{RegSc}(L)]$ which is precisely $\mathrm{C}(L)$. Indeed, the inclusion $g_{1}[\operatorname{RegSc}(L)] \subseteq \mathrm{C}(L)$ follows from (1.1); for the reverse inclusion, given an $f \in \mathrm{C}(L)$, take the $\sigma_{f}$ of (1.2) and then the corresponding regular scale $\sigma_{f}^{* *}$, which also induces the given $f$.

$\left(f_{2}\right)$ : This case can be proved in a similar way by taking $f_{2}: \operatorname{NLSC}^{c b}(L) \rightarrow$ $\operatorname{RegGSc}{ }^{c b}(L)$ defined by $f_{2}(h)(q)=h_{q}{ }^{* *}$ for every $h \in \operatorname{NLSC}^{c b}(L)$ and $q \in$ $\mathbb{Q}$, where each $h_{q}$ is given by the identity $h(q,-)=\mathfrak{c}\left(h_{q}\right)$. The identity $g_{2}(\operatorname{RegSc}(L))=\mathrm{C}(L)$ follows as in the previous case.

$\left(f_{3}\right)$ : This can be also proved similarly by taking, as in the preceding case, $f_{3}: \mathrm{H}^{c b}(L) \rightarrow \operatorname{RegGSc}^{c b}(L)$ defined by $f_{2}(h)(q)=h_{q}{ }^{* *}$ for every $h \in \mathrm{H}^{c b}(L)$ and $q \in \mathbb{Q}$, where each $h_{q}$ is given by the identity $h(q,-)=\mathfrak{c}\left(h_{q}\right)$. The identity $g_{3}(\operatorname{RegSc}(L))=\mathrm{C}(L)$ may be checked similarly as in the first case.

In summary, we have the following diagram

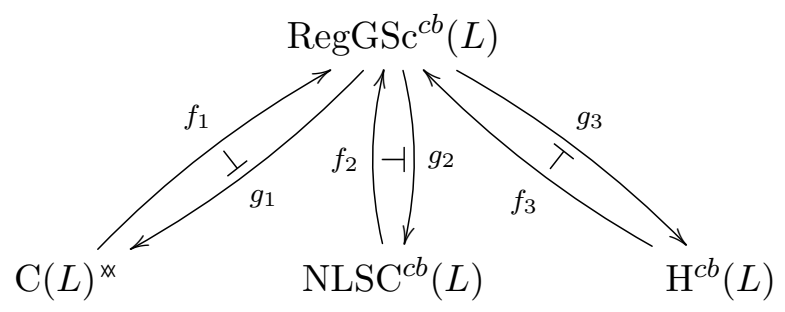

where each pair of Galois adjoint maps satisfies the conditions of Theorem 1.1 whenever $L$ is completely regular. Hence, Theorem 1.1 yields the following:

Corollary 4.2. Let $L$ be a completely regular frame. Each one of the lattices $\mathrm{C}(L)^{x}, \operatorname{NLSC}^{c b}(L)$ and $\mathrm{H}^{c b}(L)$ is (isomorphic to) the Dedekind completion of $\mathrm{C}(L)$.

Remark 4.3. Obviously, Proposition 6.1 from [2] provides another possible representation of the completion (with the additional feature that avoids either sublocales and partial reals). Indeed, recall the frame $\mathfrak{L}_{u}(\mathbb{R})$ of upper reals, that is, the subframe of $\mathfrak{L}(\mathbb{R})$ given just by generators $(p,-)$ and relations 
(R1) and (R3). In view of [2, Prop. 6.1], there is an isomorphism between the lattice $\operatorname{LSC}(L)$ of lower semicontinuous functions in $L$ and the lattice of all frame homomorphisms $h: \mathfrak{L}_{u}(\mathbb{R}) \rightarrow L$ such that $\bigvee_{r \in \mathbb{Q}} \mathfrak{o}(h(r,-))=1$. The restriction of this isomorphism to $\operatorname{NLSC}^{c b}(L)$ takes values in the set consisting of all continuously bounded frame homomorphisms $\mathfrak{L}_{u}(\mathbb{R}) \rightarrow L$ such that $h(p,-) \geqslant h(r,-)^{* *}$ for all $p<r$ (that we shall denote by $\left.\operatorname{nlsc}^{c b}(L)\right)$.

The Galois connection between $\operatorname{RegGSc}^{c b}(L)$ and $\operatorname{nlsc}^{c b}(L)$ is easily defined:

- $g_{4}(\sigma): \mathfrak{L}_{u}(\mathbb{R}) \rightarrow L$ is the frame homomorphism defined on generators by

$$
g_{4}(\sigma)(p,-)=\bigvee_{r>p} \sigma(r) .
$$

- $f_{4}: \operatorname{nlsc}^{c b}(L) \rightarrow \operatorname{RegGSc} c^{c b}(L)$ is defined by $f_{4}(h)(q)=h(q,-)^{* *}$ for each $h \in \operatorname{nlsc}^{c b}(L)$ and $q \in \mathbb{Q}$.

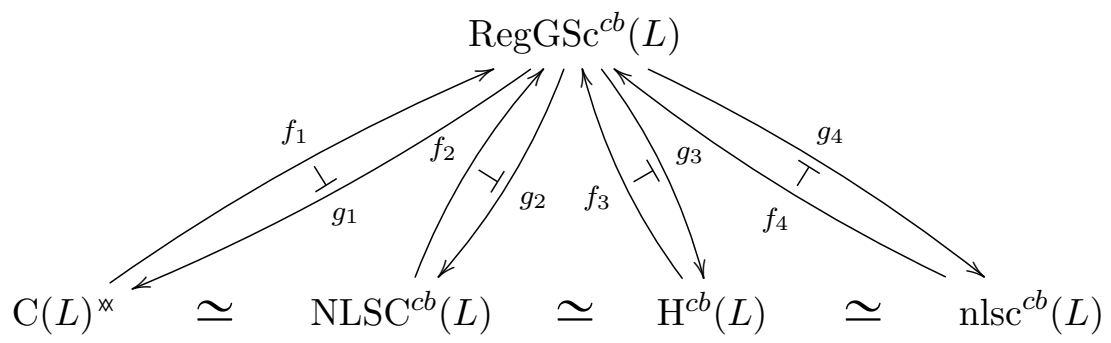

Figure 1. The unified picture.

\section{A CLOSing REMARK}

We conclude the paper with a new simpler proof of [7, Proposition 3.1], that is inspired by Proposition 3.3 and does not require the use of the lattice ordered ring structure of $\mathrm{C}(L)$.

Proposition 5.1. Let $L$ be a completely regular frame and let $h \in \operatorname{IC}(L)$ be such that

(1) $\{f \in \mathrm{C}(L) \mid f \leqslant h\} \neq \varnothing$, and

(2) $h(p,-)^{*} \leqslant h(-, q)$ whenever $p<q$.

Then $h=\bigvee^{\mathrm{IC}(L)}\{f \in \mathrm{C}(L) \mid f \leqslant h\}$.

Proof. Let $\mathcal{F}=\{f \in \mathrm{C}(L) \mid f \leqslant h\}$. By $(1), \mathcal{F} \neq \varnothing$. Since $\operatorname{IC}(L)$ is Dedekind complete, the supremum $f_{\vee}=\bigvee^{\mathrm{IC}(L)} \mathcal{F}$ exists. We shall prove that $f_{\vee}=h$.

For this purpose, fix a $q \in \mathbb{Q}$ and an $a \in L$ such that $a \ll h(q,-)$. Then, by the complete regularity of $L$, there exists a family $\left\{c_{r} \in L \mid r \in \mathbb{Q} \cap[0,1]\right\}$ 
such that $a \leqslant c_{0}, c_{1} \leqslant h(q,-)$ and $c_{r}<c_{s}$ whenever $r<s$. Furthermore, let $\psi_{q}: \mathbb{Q} \cap(-\infty, q] \rightarrow \mathbb{Q} \cap[0,1)$ be a dual-order isomorphism. Then, for each $f \in \mathcal{F}$ define the mapping $\sigma_{q, a}: \mathbb{Q} \rightarrow L$ by

$$
\sigma_{q, a}(r)= \begin{cases}f(r,-) & \text { if } r>q \\ f(r,-) \vee c_{\psi_{q}(r)} & \text { if } r \leqslant q .\end{cases}
$$

Notice that $\bigvee_{r \in \mathbb{Q}} \sigma_{q, a}(r) \geqslant \bigvee_{r \in \mathbb{Q}} f(r,-)=1$. Moreover

$$
\bigvee_{r \in \mathbb{Q}} \sigma_{q, a}(r)^{*} \geqslant \bigvee_{r \in \mathbb{Q}} h(r,-)^{*} \geqslant \bigvee_{r \in \mathbb{Q}} h(-, r)=1,
$$

since $\sigma_{q, a}(r) \leqslant h(r,-)$ for all $r \in \mathbb{Q}$. Note further that $f(s,-)<f(r,-)$ and $c_{\psi_{q}(s)}<c_{\psi_{q}(r)}$ for every $r<s$ in $\mathbb{Q}$. Consequently, $\sigma_{q, a}(s)<\sigma_{q, a}(r)$ for every $r<s$ and thus $\sigma_{q, a}$ is a scale that determines a continuous real function $f_{q, a}$ via formulas (1.1). It is easy to check that $f_{q, a} \in \mathcal{F}$ and consequently that $f_{\vee} \geqslant f_{q, a}$. Hence, by the complete regularity of $L$, we have

$$
f_{\vee}(q,-) \geqslant \bigvee_{a \ll h(q,-)} f_{q, a}(q,-) \geqslant \bigvee_{a \ll h(q,-)} a=h(q,-)
$$

for each $q \in \mathbb{Q}$. Furthermore, using (2) it follows that $h(-, q) \geqslant h(p,-)^{*} \geqslant$ $f_{\vee}(p,-)^{*} \geqslant f_{\vee}(-, p)$ for every $p<q$ in $\mathbb{Q}$. Then, finally,

$$
f_{\vee}(-, q)=\bigvee_{p<q} f_{\vee}(-, p) \leqslant h(-, q)
$$

for every $q \in \mathbb{Q}$.

\section{REFERENCES}

[1] B. Banaschewski, The real numbers in pointfree topology, Textos de Matemática, vol. 12, Universidade de Coimbra (1997).

[2] J. Gutiérrez García, T. Kubiak and J. Picado, Localic real-valued functions: a general setting, J. Pure Appl. Algebra 213 (2009) 1064-1074.

[3] J. Gutiérrez García, I. Mozo Carollo and J. Picado, Normal semicontinuity and the completion of pointfree function rings, Algebra Universalis, doi: 10.1007/s00012-0160378-z, to appear.

[4] J. Gutiérrez García and J. Picado, Rings of real functions in pointfree topology, Topology Appl. 158 (2011) 2264-2278.

[5] J. Gutiérrez García and J. Picado, On the parallel between normality and extremal disconnectedness, J. Pure Appl. Algebra 218 (2014) 784-803.

[6] I. Mozo Carollo, Frame presentations: variants of the reals, rings of functions, their Dedekind completions, and the unit circle, PhD Thesis, University of the Basque Country, Bilbao, 2015.

[7] I. Mozo Carollo, J. Gutiérrez García and J. Picado, On the Dedekind completion of function rings, Forum Math. 27 (2015) 2551-2585.

[8] J. Picado and A. Pultr, Frames and locales: Topology without points, Frontiers in Mathematics, vol. 28, Springer, Basel, 2012. 
[9] S. A. Steinberg, Lattice-ordered rings and modules, Springer, New York (2010).

Department of Mathematics, University of the Basque Country UPV/EHU, Apdo. 644, 48080 Bilbao, Spain

E-mail address: javier.gutierrezgarcia@lg.ehu.eus

$U R L$ : www. ehu.eus/javiergutierrezgarcia

Department of Mathematics, University of the Basque Country UPV/EHU, Apdo. 644, 48080 BilbaO, SpAin

E-mail address: imanol.mozo@ehu.eus

CMuC, Department of Mathematics, University of Coimbra, 3001-501 CoimBRA, Portugal

E-mail address: picado@mat.uc.pt

$U R L:$ www.mat.uc.pt/ ${ }^{\text {picado }}$ 